\title{
Activity/inactivity circadian rhythm shows high similarities between young obesity-induced rats and old rats
}

\author{
R Bravo Santos ${ }^{1}$, J Delgado ${ }^{2}$, J Cubero ${ }^{1,3}$, L Franco ${ }^{1}, \mathbf{S}$ Ruiz-Moyano ${ }^{4}$, M Mesa ${ }^{1}$, \\ AB Rodríguez ${ }^{1}, C$ Uguz $^{5}, C$ Barriga ${ }^{1}$
}

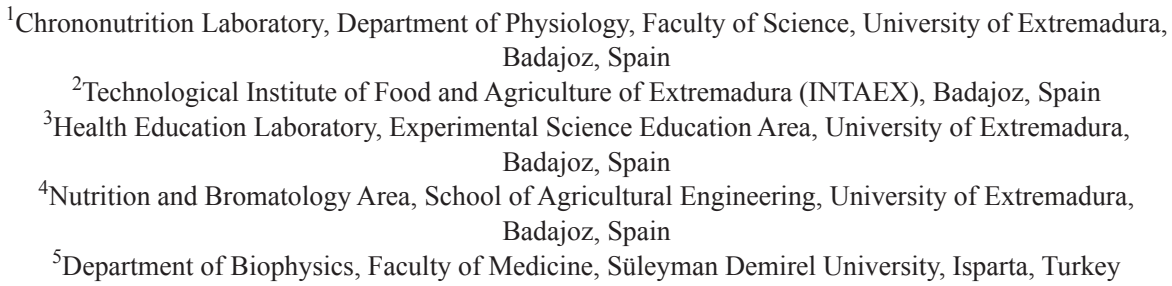

Received: July 31, 2014

Accepted: December 9, 2015

\begin{abstract}
The objective of the present study was to compare differences between elderly rats and young obesity-induced rats in their activity/inactivity circadian rhythm. The investigation was motivated by the differences reported previously for the circadian rhythms of both obese and elderly humans (and other animals), and those of healthy, young or mature individuals. Three groups of rats were formed: a young control group which was fed a standard chow for rodents; a young obesity-induced group which was fed a high-fat diet for four months; and an elderly control group with rats aged 2.5 years that was fed a standard chow for rodents. Activity/inactivity data were registered through actimetry using infrared actimeter systems in each cage to detect activity. Data were logged on a computer and chronobiological analysis were performed. The results showed diurnal activity (sleep time), nocturnal activity (awake time), amplitude, acrophase, and interdaily stability to be similar between the young obesity-induced group and the elderly control group, but different in the young control group. We have concluded that obesity leads to a chronodisruption status in the body similar to the circadian rhythm degradation observed in the elderly.
\end{abstract}

Keywords: chronobiology, chronodisruption, actimetry, rats, obesity, elderly

Rhythmic oscillations are present in numerous physiological functions (23). Human homeostatic systems are adapted to daily environmental changes provoked by the Earth's rotation in such a way that the body anticipates sleep and activity periods (21). All organisms maintain a large number of physiological functions such as the sleep/wake cycle, locomotor activity, temperature regulation, and circulating hormones, among others, under the control of the endogenous circadian clock (2). The sleep/wake circadian rhythm is a crucial process for maintaining a healthy lifestyle due to the fact that many processes such as immune system re-establishment, neural repair and resting take place during sleep time. (3).

Corresponding author: Rafael Bravo

Department of Physiology, Faculty of Science, University of Extremadura

Avda. de Elvas, s/n, 06006, Badajoz, Spain

Phone: +34 924289 388; Fax: +34 924289 388; E-mail: rbravo@unex.es 
It is well known that circadian rhythms in the elderly (both humans and other animals) present several impairments. These populations suffer from lower amplitude, advanced phase, longer sleep onset and greater locomotor activity, and lower sleep efficiency with respect to young individuals $(7,30,39)$. These circadian rhythm impairments have been found in other pathologies such as Alzheimer's disease, depression, schizophrenia, vascular disorders, metabolic syndrome, and obesity, among others $(7,19,23,47)$.

The suprachiasmatic nucleus (SCN) is considered to be the master clock of vertebrates, and sets the timing of functions which follow circadian rhythms through both neural and humoral messengers such as melatonin (synthesized in the pineal gland) and neurotransmitter GABA ( $\gamma$-aminobutyric acid), together with other molecules $(11,18,44)$. Among others, endocrine organs are regarded as peripheral oscillators. These have their own circadian clocks, and are coordinated by the SCN. Some examples are the liver with the circadian biosynthesis of cholesterol, and the pancreas, which is involved in the circadian rhythm of glucose regulation (28).

Complications of obesity include high body mass index, cardiovascular disease, endothelial dysfunction, impaired glucose tolerance, type- 2 diabetes mellitus, and dyslipidæmia, among others. Previous ways of combating excess body weight have failed, and new approaches need to be taken (21). One of the possibilities lies in the field of chronobiology: obesity has been studied from a chronobiological perspective in the last few years, and several important changes in circadian rhythms have been found to join it (14). Indeed, white adipose tissue shows a circadian rhythm in its own active metabolism (5). Previous studies have reported the possibility of alterations in the biological rhythms of adipose tissue in cases of obesity or metabolic syndrome due to changes in hormonal action $(26,27)$. White adipose tissue hormones actuate circadian metabolism, endowing adipose tissue with a peripheral oscillator role with consequences for other circadian biorhythms (24).

The objective of the present study was to evaluate if the issue of induced obesity in young rats can affect their activity/inactivity circadian rhythm, and to compare any such changes with locomotor activity circadian patterns in elderly rats.

\section{Materials and Methods}

\section{Animals}

To perform the experiment, 32 four-month-old adult male Wistar rats were separated into two groups - a young control group $(\mathrm{n}=16)$ and an induced-obesity group $(\mathrm{n}=16)$. A third group, the elderly control group, was formed with 12 two-and-a-half-year-old rats whose body weights were not taken into account. The animals were individually housed under controlled environmental conditions at $24 \pm 1{ }^{\circ} \mathrm{C}$, with a $12 \mathrm{~h} / 12 \mathrm{~h}$ light-dark photoperiod.

The study was approved by the Bioethical Committee of the University of Extremadura (Badajoz, Spain) in accordance with the National Institutes of Health Guide for the Care and Use of Laboratory Animals and the European Community's Council Directives (86/609/ EEC).

\section{Animal diets}

Both the young control group and the elderly control group were fed A control maintenance chow for rodents (A04, SAFE: Scientific Animal Food \& Engineering()). The induced- 
obesity group was fed the Purified Diet $230 \mathrm{HF}$ made by SAFEC (Vannes, France), the composition of which is presented in Table I. Every group was fed ad libitum (food and water).

Table 1

\begin{tabular}{|l|c|c|}
\hline \multicolumn{1}{|c|}{ Nutrients } & Standard diet & Hypercaloric diet \\
\hline Proteins & $\mathbf{\%}$ & $\mathbf{\%}$ \\
\hline Lipids & 16 & 20.5 \\
\hline Carbohydrates & 3 & 35 \\
\hline Fibre & 47.8 & 36.8 \\
\hline Minerals and vitamins & 3.4 & - \\
\hline
\end{tabular}

Diets used in the assay: Standard diet (A04, SAFEC) used to feed both young and old control groups, and a hypercaloric diet (Purified diet 230F, SAFE(C) used to elicit obesity in the treatment group.

Non-specified percentages are due to moisture.

\section{Activity measure}

Each cage was equipped with an infrared actimeter system to detect activity using two crossed perpendicular infrared beams situated on a plane $70 \mathrm{~mm}$ above the sustenance plane. Motor activity data for each cage were automatically logged every 15 min onto a single personal computer, and analysed every 7 days for all the different experimental groups using the program DAS 24C (Barcelona, Spain) (21). For the statistical calculations and the mathematical modeling, the data were binned into hourly intervals.

Rhythmicity was measured with the software (Ritme $\mathbb{C}$, Barcelona, Spain) which performs a cosinor analysis fitting a sinusoidal function to the experimental variables. The mathematical expression used is:

$$
\mathrm{Y}=\mathrm{M}+\mathrm{A} \cos (\omega \mathrm{t}-\varphi)
$$

where M is the MESOR (Midline-Estimating Statistic Of Rhythm), A is the amplitude of the function, $\omega$ is the angular frequency $2 \pi / T$ (where $T$ is the period, in the present case $T=24 \mathrm{~h}$ ), and $\varphi$ is the acrophase.

\section{Non-parametric circadian rhythm variables}

The interdaily stability (IS) quantifies the invariability between days, thus providing an indication of the strength of coupling of the rhythm to the supposedly stable environmental zeitgeber. It is calculated as the ratio between the variance of the average 24-hour pattern around the mean and the overall variance:

$$
I S=\frac{\mathrm{n} \sum_{h=1}^{P}\left(\bar{x}_{h}-\bar{x}\right)^{2}}{\mathrm{p} \sum_{i=1}^{n}\left(x_{i}-\bar{x}\right)^{2}}
$$

where $n$ is the total number of data, $p$ is the number of data per day (in this assay 24), are the hourly means, is the mean of all data, and represents the individual data points. 
The intradaily variability (IV) gives an indication of the fragmentation of the rhythm, i.e., the frequency of and extent of transitions between rest and activity and is calculated as the ratio of the mean squares of the differences between successive hours (first derivative) and the mean squares around the grand mean (overall variance).

$$
I V=\frac{\mathrm{n} \sum_{i=2}^{n}\left(x_{i}-x_{i-1}\right)^{2}}{(\mathrm{n}-1) \sum_{i=1}^{n}\left(x_{i}-\bar{x}\right)^{2}}
$$

For the statistical analysis of the data, the software package Graphpad Prism v. 5.02@ (La Jolla, USA) was used. Two types of study were carried out:

1. Descriptive, calculating as representative values of the arithmetic mean \pm standard error.

2. Hypothesis testing. Because of the available sample size, a Kolmogorov-Smirnov test was applied to check the normality of the study variables. The results were analysed using Student's $t$-test or the Mann-Whitney U-test, with the significance level taken to be $p<0.05$. To elucidate which experimental group could have significant differences Dunn's Post Hoc was performed when it was required.

\section{Results}

After four months, the young obesity-induced rats had a greater body weight than the young control rats $(\mathrm{p}<0.001)$, as can be seen in Fig. 1.

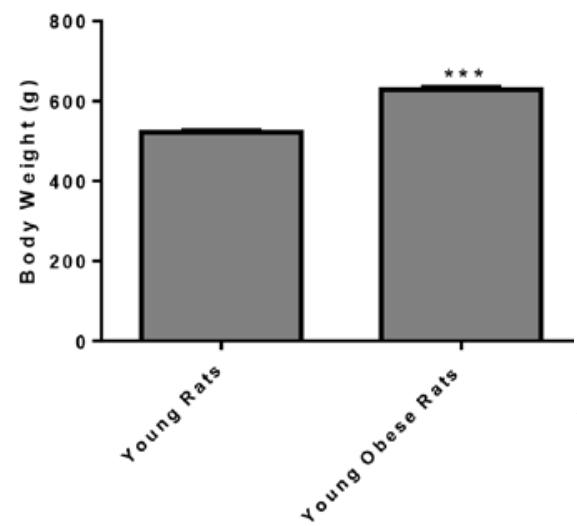

Fig 1. Body weight (Mean $\pm \mathrm{SEM}$ ) comparison between young control rats $(525.1 \pm 6.135 \mathrm{~g} ; \mathrm{n}=16)$ and high-fat fed rats $(629.1 \pm 6.01 \mathrm{~g} ; \mathrm{n}=16)$. Mann-Whitney U-Test was performed. $* * * \mathrm{p}<0.001$

The rats' diurnal activity (sleep time) is represented in Fig. 2 which shows high activity both in the young obesity-induced group $(\mathrm{p}<0.001)$ and in the elderly control group $(\mathrm{p}<$ 0.001 ) in contrast with the young, control rats. Also, the obesity-induced rats have increased their sleep activity when compared to the elderly rats $(\mathrm{p}<0.05)$.

Nocturnal locomotor activity was similar in the young obesity-induced rats and the elderly control group (Fig. 2). Moreover, these groups had lower values of activity ( $\mathrm{p}<$ 0.001 ) than the young control group during the awake period.

Regarding chronobiological parameters, the MESOR (A), the amplitude (B) and the acrophase (C) are represented in Fig. 3. MESOR did not show any statistical difference amid any group. Amplitude and acrophase showed lower values when comparing both elderly control rats and obesity-induced rats $(\mathrm{p}<0.001$ in both groups' amplitude, and $\mathrm{p}<0.05$ in both groups' acrophase). 


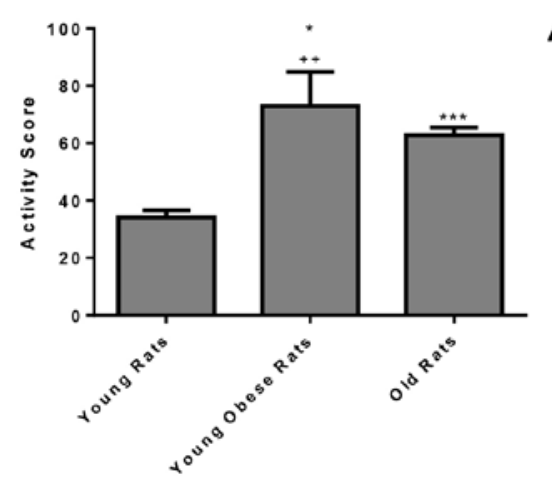

A

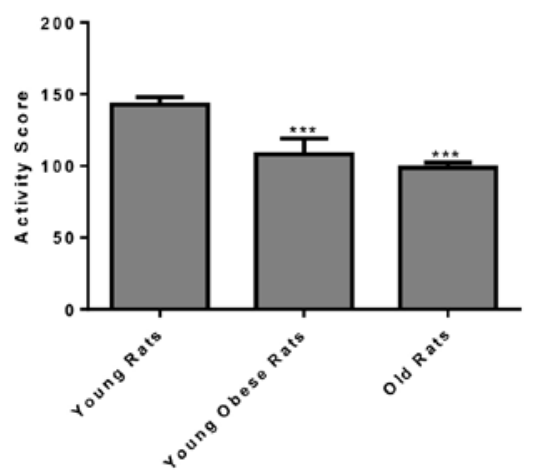

Fig. 2. Locomotor activity representation (activity pulses per hour: Mean \pm SEM) during the week of the study in young control rats $(n=16)$, obese-like rats $(n=16)$ and old control rats $(n=12)$.

Kruskal-Wallis test and Dunn's post-hoc were performed

A) Diurnal activity - activity during the rest period -. ${ }^{*} \mathrm{p}<0.05$ young control rats $(34.23 \pm 2.4 ; \mathrm{n}=16)$

vs. obese-like rats $(72.99 \pm 2.6 ; \mathrm{n}=16) ; * * * \mathrm{p}<0.001$ young control rats vs. old control rats

$(62.86 \pm 11.8 ; \mathrm{n}=12) ;+\mathrm{p}<0.01$ obese-like rats vs. old control rats.

B) Nocturnal activity - activity during the wakefulness period-.

$* * * \mathrm{p}<0.001$ young control rats $(142.57 \pm 5.4 ; \mathrm{n}=16)$ vs. both old control rats $(98.80 \pm 11.10 ; \mathrm{n}=16)$ and obese-like rats $(108.03 \pm 3.36 ; \mathrm{n}=12)$.

A

M ESOR

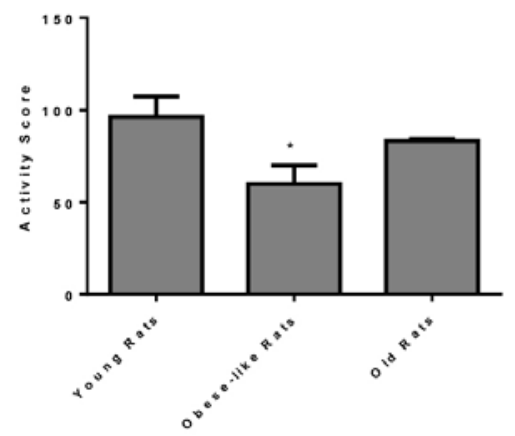

C






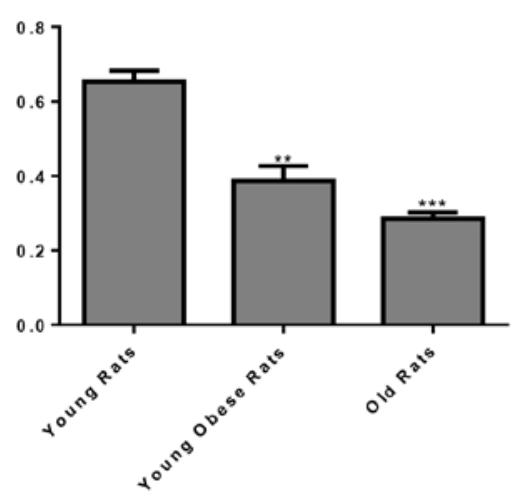

A

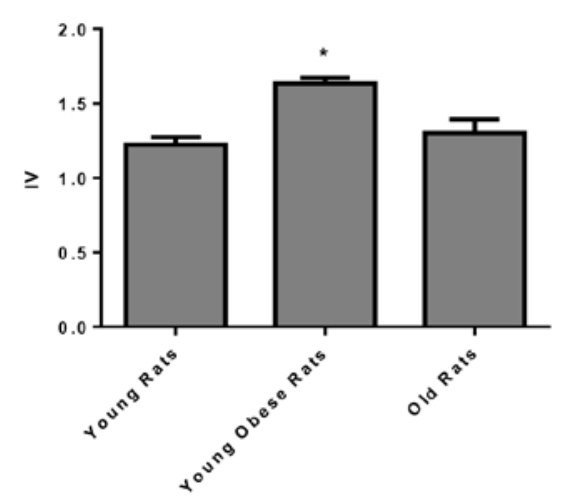

Fig. 4. Non-parametric circadian rhythms variables representation (Mean $\pm \mathrm{SEM}$ ) during the week of the study. Kruskal-Wallis test and Dunn's post-hoc were performed.

A) Interdaily stability - rhythm constancy $-* * p<0.01$ young control rats $(0.65 \pm 0.03 ; n=16)$ vs. high-fat fed rats $(0.28 \pm 0.02 ; \mathrm{n}=16) . * * * \mathrm{p}<0.001$ young control rats vs. old control rats $(0.38 \pm 0.04 ; \mathrm{n}=12)$.

B) Intradaily Variability - rhythm fragmentation.

$* \mathrm{p}<0.05$ young control $(1.22 \pm 0.05 ; \mathrm{n}=16)$ rats vs. high-fat fed rats $(1.30 \pm 0.09 ; \mathrm{n}=16)$.

Figure 4 shows the Interdaily Stability results. Both the old control group and the obesity-induced group presented lower values of this parameter than the young control group $(\mathrm{p}<0.05)$. For the Intraday Variability (Fig. 4), only the elderly control group showed differences compared to the young control group $(\mathrm{p}<0.05)$.

\section{Discussion}

Ageing is a very complex process that shows symptoms of chronodisruption that produces sleep disturbances (30) and other physiological or psychological dysfunctions (2), including impaired nutrient absorption $(37,47)$, immunosenescence $(9,31,48)$, decrease in hormone levels (38), and neuronal cell death $(20,40)$. The elderly rats used in our research showed such chronodisruption symptoms as an increase in diurnal activity (sleep time) and intraday variability in locomotor activity. There were also decreases in nocturnal activity (awake time), amplitude, and interday stability, and an advance in phase (acrophase) in contrast with the young control rats. These locomotor alterations have been correlated with lower serotonin and melatonin levels, widely accepted as changes that occur with ageing. Indeed, the increase of the neurotransmitter's levels of serotonin or indole melatonin is capable of reconsolidate the sleep/wake cycle in elderly humans and animals $(25,39)$.

Locomotor activity decreases, in obesity, have been appointed classically as consequences provoked by a reduction in muscle mass, which may be substituted by adipose tissue without changes in body weight. In addition, Bartol-Munier and colleagues (4) have reported that both locomotor activity and interdaily stability drop during wakefulness due to diet, when a high-fat diet is administered to get obese animals. Moreover, these changes were confirmed by our research group previously in a similar essay carried out in animals which were fed a high-fat diet (6). 
White adipose tissue is not only an energy store but also an active endocrine organ which produces hormones and bioactive peptides, known as adipokines, which act at both the autocrine/paracrine and endocrine levels (15). In recent years, it has been reported that some functions of adipose tissue follow a rhythmic pattern which give to this tissue the role of peripheral oscillator $(29,32)$. Leptin hormone is produced by adipose tissue as a function of fat stores, i.e., after release by the adipose tissue, leptin provides signals to the brain giving information about the status of the body's energy stores (43). In obese individuals, a physiological status of leptin resistance has been reported. Moreover, alterations in leptin signalization are implicated in sleep/wake cycle alterations, so that they may represent an important link between metabolic regulation and sleep. Indeed, ob/ob (leptin-deficient) mice show an unconsolidated sleep/wake cycle (33). It has been shown that both leptin deficiency and high leptin levels are related to locomotor activity, sleep disturbances, and the hormone ghrelin: in obesity, high leptin levels provoke leptin-resistance - a decrease in the sleeppromoting hormone effect - and an increase in the levels of ghrelin, a hunger hormone which increases with sleep restriction $(14,35)$.

Recently, it has been suggested that high-fat diets in rats produce several changes in the expression of circadian-clock genes. Clock genes are present in all tissues, and their products interact reciprocally both at the transcriptional and at the translational levels to produce circadian oscillations (28). The CLOCK and BMAL1 proteins (which have the basic helixloop-helix functional domain, providing them with a DNA-binding capacity) form a heterodimer through the PAS domain comprising the positive limb of the circadian oscillator (10) to activate the E-box-mediated transcription of Per and Cry genes as negative elements repressing the transcriptional activity of CLOCK:BMAL1 (1). In addition, Rev-erb $\alpha$ and ROR $\alpha$, another two E-box-mediated genes, repress and activate Bmall transcription, respectively (16). Once PER and CRY reach a critical level, they enter into the nucleus to inhibit the transactivation mediated by the CLOCK:BMAL1 complex, i.e., PER and CRY inhibit their own transcription $(17,21)$. Casein kinase I epsilon and delta $(\mathrm{CKI} \varepsilon / \delta)$ phosphorylation of the PER and CRY proteins promotes their proteasomal degradation, an important step in the generation of circadian rhythmicity (22). It has been proposed that highfat diets are able to increase CKI $\varepsilon$ activity and lead to mPER2 degradation, thus provoking an advance in phase of the circadian expression pattern of clock genes (21).

The results of the present study have shown a reduced amplitude and an advanced phase in the locomotor activity/inactivity circadian rhythm of young obesity-induced rats and elderly control rats in relation with the young control group. In addition, lower nocturnal activity and greater diurnal activity were observed in the elderly control and the obesityinduced groups compared to the young control group. Previous studies have reported that obesity-induced mice have an altered circadian genetic expression when compared to control groups. An advanced phase in liver and lower amplitude in adipose tissue is caused by Rev-erb $\alpha$ alterations, whose action is affected by consumption of a high-fat diet ( $45 \%$ fat) in control mice (36). While such alterations are chronodisruption symptoms, which are associated with elderly individuals, our research group has also found them in young inducedobesity rats. One can thus consider that obesity is able to modify the body's rhythmic physiological patterns, producing the same alterations that appear in the elderly.

Finally, a loss of circadian rhythm consistency was observed in the obesity-induced and the elderly rats as was shown by the interdaily stability, although the induced-obesity rats showed no statistically significant changes in Intradaily Variability whereas both of these parameters are impaired in ageing (30). This visible link between chronodisruption in the 
elderly and obesity-induced subject is coherent with previous studies carried out at molecular levels (34). To sum up, a high-fat diet may provoke a chronosenescence status in the biological rhythms of young individuals.

To support the link between old chronotype and obesity-induced ones, we have found there are not many references in scientific literature establishing similarities between obesity and aging: several research have been carried out in this field with interesting results. These researches have been mainly focused on immunity, like proinflammatory variables measured in gene expression - cellular senescence - (45), natural killer activity (12), lymphocyte proliferation (13) strengthening in this way the concept of immunosenescence (38). From a non-immunity point of view, some researches have reported that obesity leads to a premature senescence in heart (41) and in vascular endothelium $(8,47)$; even, inflammation due to body weight excess has been related with erosion of telomeres in DNA (42) as it occurs in aging.

The present results have shown that the induced-obesity activity/inactivity chronotype presents major similarities with those of old individuals, and that both of these groups differ from young individuals in their sleep/wake circadian rhythm patterns. The findings add further support to the idea that epigenetic methods, such as healthy diets based on chrononutrition principles or daily physical exercise may contribute to reducing body weight and re-consolidating circadian rhythms.

\section{REFERENCES}

1. Allebrandt KV, Roenneberg T (2013): Gene variants associated with sleep durations: implications for metabolic dysfunction. In: Handbook of Nutrition, diet and sleep, eds Preedy VR, Patel VB, Le L. Wageningen Academic Publishers, Wageningen, pp. 72-87

2. Barnard AR, Nolan PM: When clocks go bad: neurobehavioural consequences of disrupted circadian timing. PloS Genet. 4, e1000040 (2008)

3. Barriga C, Rodríguez AB, Esteban S, Rial RV: Interrelaciones entre el sueño y el estado immune (Interrelations between sleep and the immune status). Rev. Neurol. 40, 548-556 (2005)

4. Bartol-Munier I, Gourmelen S, Pevet P, Challet E: Combined effects of high-fat feeding and circadian desynchronization. Int. J. Obesity. 30, 60-67 (2006)

5. Beccuti G, Pannain S: Sleep and obesity. Curr. Opin. Clin. Nutr. Metab. Care. 14, 402-412 (2011)

6. Bravo R, Matito S, Cubero J, Paredes SD, Franco L, Rivero M, Rodríguez AB, Barriga C: Tryptophan-enriched cereal intake improves nocturnal sleep, melatonin, serotonin, and total antioxidant capacity levels and mood in elderly humans. Age 35, 1277-1285 (2013)

7. Bravo R, Cubero J, Franco L, Mesa M, Galán C, Rodríguez AB, Jarne C, Barriga C: Body weight gain in rats by a high-fat diet produces chronodisruption in activity/inactivity circadian rhythm. Chronobiol. Int. 31, 363-70 (2014)

8. Brodsky SV, Gealekman O, Chen J, Zhang F, Togashi N, Crabtree M, Gross SS, Nasjletti A, Goligorsky MS: Prevention and reversal of premature endothelial cell senescence and vasculopathy in obesity-induced diabetes by ebselen. Circ. Res. 94, 377-384 (2004)

9. Canizzo ES, Clement CC, Sahu R, Follo C, Santambrogio L: Oxidative stress, inflamm-aging and immunosenescence. J. Proteome 74, 2313-2323 (2011)

10. Chapnik N, Solomon G, Genzer Y, Miskin R, Gertler A, Froy O: A superactive leptin antagonist alters metabolism and locomotion in high-leptin mice. J. Endocrinol. 217, 283-290 (2013)

11. Cubero J, Narciso D, Terrón MP, Rial R, Esteban S, Rivero M, Parvez H, Rodríguez AB, Barriga C: Chrononutrition applied to formula milks to consolidate infants' sleep/wake cycle. Neuroendocrinol. Lett. 28, 360-366 (2007)

12. De Castro N, Vida C, Hernández O, Cruces J, De la Fuente M: Lymphocyte proliferation and natural killer activity in young genetically obese mice. Ann. Nutr. Metab. 58, 243 (2011)

13. De la Fuente M, Hernández O, Causape S, Gheorghe A, Díaz L, Marcos A: Adult diet-obese mice show a premature senescence in natural killer activity. Ann. Nutr. Metab. 58, 253 (2011) 
14. Delezie J, Challet E: Interactions between metabolism and circadian clocks: reciprocal disturbances. Ann. N. Y. Acad. Sci. 1243, 30-46 (2011)

15. Delgado J, Terrón MP, Garrido M, Pariente JA, Barriga C, Rodríguez AB, Paredes SD: A cherry nutraceutical modulates melatonin, serotonin, corticosterone, and total antioxidant capacity levels: effect on ageing and chronotype. J. Appl. Biomed. 10, 109-117 (2012)

16. Demarque M, Schibler U: Shedding new light on circadian clocks. eLife. 2, e00659 (2013)

17. Duez H, Staels B: Rev-erv- $\alpha$ : an integrator of circadian rhythms and metabolism. J. Appl. Physiol. 107, 19721980 (2009)

18. Duncan MJ, Congleton MR: Neural mechanisms mediating circadian phase resetting by activation of 5-HT7 receptors in the dorsal raphe: roles of GABAergic and glutamatergic neurotransmission. Brain Res. 1366, 110 119 (2010)

19. Ferrari E, Magri F: Role of neuroendocrine pathways in cognitive decline during aging. Ageing Res. Rev. 7 , 225-233 (2008)

20. Franco L, Sánchez CL, Bravo R, Rodríguez AB, Barriga C, Cubero J: The sedative effects of hops (Humulus lupulus), a component of beer, on the activity/rest rhythm. Acta Physiol. Hung. 99, 133-139 (2012)

21. Froy O: Metabolism and circadian rhythms - Implications for obesity. Endocr. Rev. 31, 1-24 (2010)

22. Froy O: The circadian clock and metabolism. Clin. Sci. 120, 65-72 (2011)

23. Garaulet M, Madrid JA: Chronobiology, genetics and metabolic syndrome. Curr. Opin. Lipidol. 20, 127-134 (2009)

24. Garaulet M, Ordovás JM, Madrid JA: The chronobiology, etiology and pathophysiology of obesity. Int. J. Obesity 34, 1667-1683 (2010)

25. Garrido M, Paredes SD, Cubero J, Lozano M, Toribio-Delgado AF, Muñoz JL, Reiter RJ, Barriga C, Rodríguez AB: Jerte Valley cherry-enriched diets improve nocturnal rest and increase 6-sulfatoxymelatonin and total antioxidant capacity in the urine of middle-aged and elderly humans. J. Gerontol. A Biol. Sci. Med. Sci. 65, 909-914 (2010)

26. Gómez-Abellán P, Garaulet M: Adipose tissue as a peripheral clock. In: Chronobiology and Obesity, eds Garaulet M, Ordovás JM, Springer, New York, pp. 29-53 (2013)

27. Gómez-Abellán P, Gómez-Santos C, Madrid JA, Milagro FI, Campion J, Martínez JA, Ordovás JM, Garaulet M: Circadian expression of adiponectin and its receptors in human adipose tissue. Endocrinology 151, 115-122 (2010)

28. Gómez-Abellán P, Madrid JA, Ordovás JM, Garaulet M: Aspectos cronobiológicos de la obesidad y el síndrome metabólico (Chronobiological aspects of obesity and metabolic syndrome). Endocrinol. Nutr. 59, 50-61 (2012)

29. Gómez-Santos C, Gómez-Abellán P, Madrid JA, Hernández-Morante JJ, Luján JA, Ordovás JM, Garaulet M: Circadian rhythm of clock genes in human adipose explants. Obesity 17, 1481-1485 (2009)

30. Huang H, Liu R, Wang Q, van Someren EJW, Xu H, Zhou J: Age-associated difference in circadian sleep-wake and rest-activity rhythms. Physiol. Behav. 76, 597-603 (2002)

31. Hutt HJ, Bennerscheidt P, Thiel B, Arand M: Immunseneszenz und Impfungen im höheren Lebensalter. (Immunosenescence and vaccinations in the elderly). Med. Klin. (Munich) 105, 802-807 (2010)

32. Kershaw EE, Flier JS: Adipose tissue as an endocrine organ. J. Clin. Endocrinol. Metab. 89, 2548-2556 (2004)

33. Klok MD, Jakobsdottir S, Drent ML: The role of leptin and gherlin in the regulation of food intake and body weight in humans: a review. Obes. Rev. 8, 21-24 (2006)

34. Kohsaka A, Laposky AD, Ramsey KM, Estrada C, Joshu C, Kobayashi Y, Turek FW, Bass J: High-fat diet disrupts behavioral and molecular circadian rhythms in mice. Cell Metabol. 6, 414-421 (2007)

35. Laposky AD, Shelton J, Bass J, Dugovic C, Perrino N, Turek FW: Altered sleep regulation in leptin-deficient mice. Am. J. Physiol. Regul. Integr. Comp. Physiol. 290, 894-903 (2006)

36. Madrid JA: An introduction to chronobiology. In: Chronobiology and Obesity, eds Garaulet M, Ordovás JM, Springer, New York, pp. 11-28 (2013)

37. Meier J, Sturm A: The intestinal epithelial barrier: does it become impaired with age? Dig. Dis. 27, 240-245 (2009)

38. Ortega E, García JJ, de la Fuente M: Ageing modulates some aspects of the non-specific immune response of murine macrophages and lymphocytes. Exp. Physiol. 85, 519-525 (2000)

39. Paredes SD, Marchena AM, Bejarano I, Espino J, Barriga C, Rial RV, Reiter RJ, Rodríguez AB: Melatonin and tryptophan affect the activity-rest rhythm, core and peripheral temperatures, and interleukin levels in the ringdove: changes with age. J. Gerontol. A. Biol. Sci. Med. Sci. 64, 340-350 (2009)

40. Qureshi GA, Parvez SH (2007): Oxidative Stress and Neurodegenerative Disorders. Elsevier, Oxford, Amsterdam, The Netherlands 
41. Shankar SK: Biology of aging brain. J. Pathol. Microbiol. 53, 595-604 (2010)

42. Takahashi K, Takatsu M, Hattori T, Murase T, Ohura S, Takeshita Y, Watanabe S, Murohara T, Nagata K: Premature cardiac senescence in DahlS.Z-Lepr(fa)/Lepr(fa) rats as a new animal model of metabolic syndrome. Nagoya J. Med. Sci. 76, 35-49 (2014)

43. Tzanetakou IP, Katsilambros NL, Beteos A, Mikhailidis DP, Perrea DN: "Is obesity linked to aging?": adipose tissue and the role of telomeres. Ageing Res. Rev. 11, 220-229 (2012)

44. Van der Spek R, Kreier F, Fliers E, Kalsbeek A: Circadian rhythm in adipose white tissue. Prog. Brain Res. 199, 183-201 (2012)

45. Van Someren EJW: Circadian and sleep disturbances in the elderly. Exp. Gerontol. 35, 1229-1237 (2000)

46. Villaret A, Galitzky J, Decaunes P, Estève D, Marques MA, Sengenès C, Chiotasso P, Tchkonia T, Lafontan M, Kirkland JL, Bouloumié A: Adipose tissue endothelial cells from obese human subjects: differences among depots in angiogenic, metabolic, and inflammatory gene expression and cellular senescence. Diabetes 59, 27552763 (2010)

47. Wang CY, Kim HH, Hiroi Y, Sawada N, Salomone S, Benjamin LE, Walsh K, Moskowitz MA, Liao JK: Obesity increases vascular senescence and susceptibility to ischemic injury through chronic activation of Akt and mTOR. Sci. Signal. 2, ra11 (2009)

48. Witting W, Kwa IH, Eikelenboom P, Mirmiran M, Swaab DF: Alterations in the circadian res-activity rhythm in aging and Alzheimer's disease. Biol. Psychiatry 27, 563-572 (1990)

49. Woudstra T, Thomson ABR: Nutrient absorption and intestinal adaptation with ageing. Best Pract. Res. Clin. Gastroenterol. 16, 1-15 (2002) 